\title{
INTELLIGENT GROWTH AUTOMATON OF VIRTUAL PLANT BASED ON PHYSIOLOGICAL ENGINE
}

\author{
Qingsheng Zhu ${ }^{*}$, Mingwei Guo, Hongchun Qu, Qingqing Deng \\ College of Computer Science, Chongqing University, Chongqing 400044, P. R. China \\ * Corresponding author, Address: College of Computer Science, Chongqing University, \\ Chongqing 400044, P. R. China, Tel: +86-23-65105660, Fax: +86-23-65105660, Email: \\ qszhu@cqu.edu.cn
}

\begin{abstract}
In this paper, a novel intelligent growth automaton of virtual plant is proposed. Initially, this intelligent growth automaton analyzes the branching pattern which is controlled by genes and then builds plant; moreover, it stores the information of plant growth, provides the interface between virtual plant and environment, and controls the growth and development of plant on the basis of environment and the function of plant organs. This intelligent growth automaton can simulate that the plant growth is controlled by genetic information system, and the information of environment and the function of plant organs. The experimental results show that the intelligent growth automaton can simulate the growth of plant conveniently and vividly.
\end{abstract}

Keywords: intelligent growth automaton, intelligent physiological engine, two dimensional hierarchical automata, virtual plant

\section{INTRODUCTION}

In recent years, research on virtual plant has made great progress. Many methods and models for generating plant graphics has been proposed, for example, mended L-system (Prusinkiewice et al., 1990), IFS (Dekmo et al., 1985), reference axis technique (Blaise et al., 1998), branch matrix (Viennot et al., 1989), particle system (Reeves et al., 1985) and so on. However, it is very difficult to depict and reproduce the complicated growth of plant.

Please use the following format when citing this chapter:

Zhu, Q., Guo, M.,. Qu, H. and Deng, Q., 2009, in IFIP International Federation for Information Processing, Volume 294, Computer and Computing Technologies in Agriculture II, Volume 2, eds. D. Li, Z. Chunjiang, (Boston: Springer), pp. 1477-1486. 
The growth and development is a kind of behavior of plant life activities. It includes two aspects. One is the increase of size and weight of plant which is the growth of plant. The other is the continuously occurring of new organs called morphogenesis, which is the development of plant (Yang Shijie et al., 2000). The growth and development of plant are controlled by the genetic information system and the environment information system. Genetic information system determines the potential pattern of growth and development of plant, while the environment information system impact on the specific performance of characters. The growth and development is a very complex dynamic process (Li Hesheng et al., 2002).

The existing plant growth models are focused on computer graphics and very limited knowledge of botany are employed to generate a beautiful plant models quickly and easily, excepting for L-system and reference axis technique. Dr. Zhao Xing et al. (Zhao Xing et al., 2001) proposed a two-scale automata model based on the growth mechanism of plant, and Kyle W. Tomlinson et al. (Kyle W. Tomlinson et al., 2007) proposed a functionalstructural model for growth of clonal bunchgrasses. However, the parameters of two-scale automata model are acquired empirically. Dr. Qu Hongchun et al. (Hongchun Qu et al., 2007) proposed the intelligent physiological engine (IPE) which consists of virtual environment, two dimensional hierarchical automata (2DHA) controlling plant branch pattern, the carbohydrate balance model on the basis of ant colony system and some other key components. IPE controls and coordinates the interaction between individual agent virtual organs (IAVOs) which constitute the virtual plant, distribution and balance of carbohydrate inside plant, and the interaction of virtual plant and the virtual environment, to drive the growth and development of plant. However, 2DHA can't satisfy the demand that IPE drive the growth and development of plant. On the basis of this framework, a novel intelligent growth automaton (IGA) of virtual plant is proposed which extends the function of 2DHA and simulates the growth and development of plant controlled by genetic information system and environment information system.

\section{FRAMEWORK DESCRIPTION}

In this framework, 2DHA simulates the function of genetic information system while IPE simulates the function of environment information system and the function of IAVOs. IGA analyzes the 2DHA and uses the controlling information from 2DHA to produce the plant. At the same time, IGA sends the interaction request to IPE, and modifies the parameters of 2DHA according to the feedback information, to simulate the environment coordinating the plant growth. 
Engine

Fig.1 describes the framework of the interaction of IGA (Intelligent Growth Automaton) with 2DHA and IPE. I represents the interface between IGA and 2DHA, while II represents the interface between IGA and IPE. (1) represents the information of controlling the plant growth; (2) represents the information of structure and physiology of plant which are computed by IGA, and the information is stored in the Bi-dimensional Hash Chain; (5) represents the information of current structure and physiology of plant; 6)represents sending the information of environment and plant structure and physiology status to IPE through II. (7)represents the information of environment and function of IAVOs' feedback from IPE; (8) represents the adjustment information of structure and physiology of plant which is computed by IGA; (3) represents the information to modify parameters of 2DHA and (4) sending the modifying information to 2DHA.

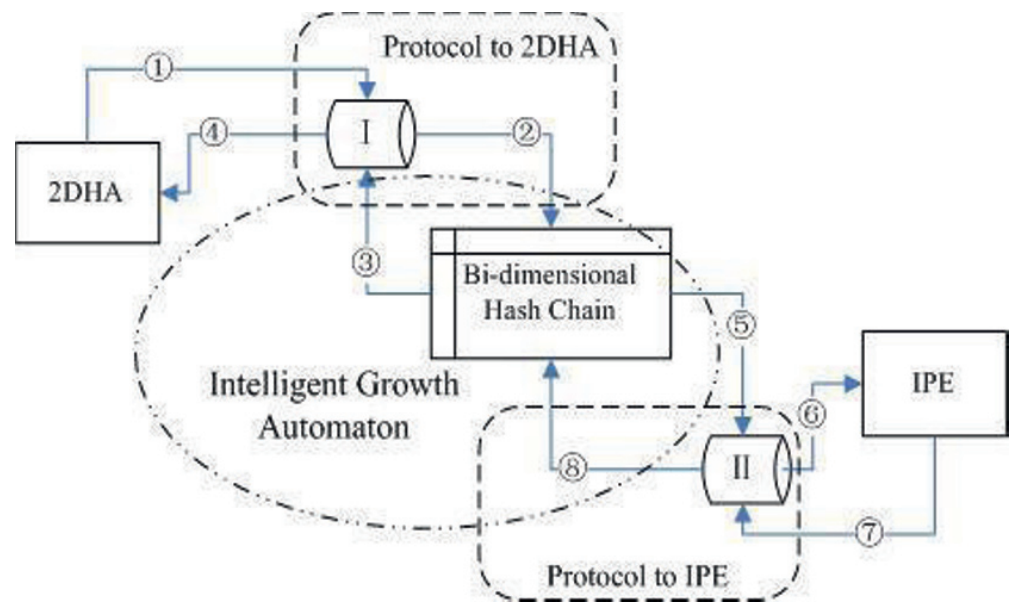

Fig. 1 Framework of Intelligent Growth Automaton

\section{THE DEVELOPMENT OF STEM}

The appearance of plant includes the stem and the organs such as leaves, flowers and fruits which grow on it. In addition, the stem is the functional organ which links the root with leaves and transport water, inorganic salt and organic nutrition (Li Hesheng et al., 2002) (Yang Shijie et al., 2000). The part where leaf grows on is called node. The part between two nodes is called internode. As shown in Fig.2, the stems constitute the main structure of the plant, bearing some buds and leafage. 


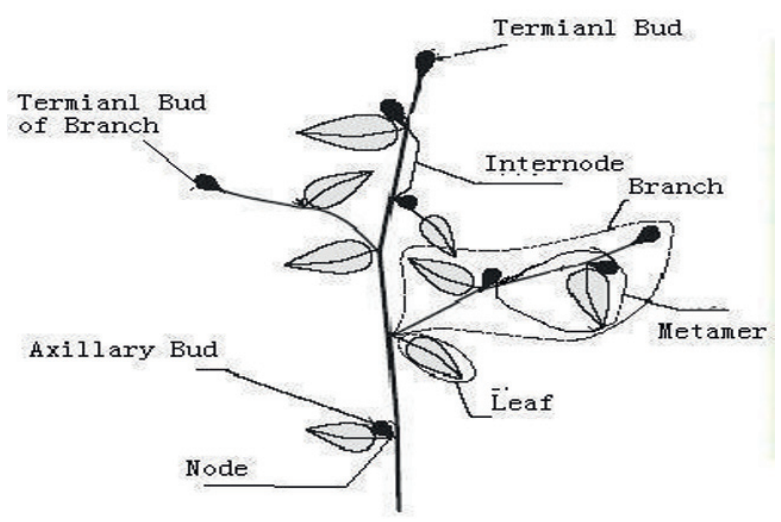

Fig. 2 Form of Stem

Metamer: it is defined as a set of internode and lateral organs (leaves, axillaries bud, flowers or fruits) between two nodes. The period of the development of a new metamer is Node Cycle.

Growth Cycle: the growth of plant is a periodical process. Each period is called a Growth Cycle, which depends on the specific plant. We assumes that all the node growth cycles are the same and the Growth Cycle of one plant could be divided into a fixed number of node growth cycles.

Growth Unit: It is the amount of new metamer in the plant axis in one Growth Cycle.

Growth Age: It is the amount of growth cycles of a plant.

The stem and flower are developed from bud. The bud at the top of the axis is called terminal bud; the bud at the leaf base is called axillary bud which develops to lateral branch. The buds are divided into active bud which grows this year and dormant bud which doesn't grow this year. The primary growth of stem includes apical growth and intercalary growth (Li Hesheng et al., 2002) (Yang Shijie et al., 2000).

\section{TWO DIMENSIONAL HIERARCHICAL AUTOMATA}

2DHA samples the plant and then acquires the parameters by employing the statistical hidden markov tree (HMT) model and clustering. 2DHA is constituted of parent automaton and child automaton. Parent automaton is constituted of parent states which transfer mutually and child automaton is constituted of child states which transfer mutually too.

Definition 1(Parent automaton): the parent automaton is defined as a finite state automaton: $A_{p}::=<Q_{p}, \pi_{p}, S_{G C}, \rho_{p}, F_{p}>$, where $Q_{p}$ denotes the 
set of parent states and a parent state represents a kind of Growth Unit. $\pi_{p}$ is the initial probability vector and used to acquire the axiom. $S_{G C}$ is the time signal to drive parent automaton and indicates time period requirement for growing one Growth Unit. $\rho_{p}$ denotes the transfer matrix of all parent states. $F_{p}$ denotes the set of terminal conditions, such as limit of plant age or a terminal parent state. It should be noted that a parent state corresponds to a child automaton.

Definition 2(Child automaton): a child automaton is a sequential automaton, it's defined as: $A_{c}::=<Q_{c}, \pi_{c}, S_{N C}, B_{c}, L_{c}, \rho_{c}, F_{c}>$.where $Q_{c}$ denotes the set of child states and a child state represents a kind of Metamer. $\pi_{c}$ denotes the initial probability of every child state when the child automaton begin to run. $S_{N C}$ is the time signal to drive child automaton and indicates time period requirement for growing one Metamer. $B_{c}$ denotes the set of axillary bud of every child state. $L_{c}$ denotes the cycle number of the child automaton continuously locating in a child state. $\rho_{c}$ denotes the transfer matrix of all child states. $F_{c}$ denotes the set of terminal conditions, such as limit of Growth Cycle or a terminal child state.

When the child automaton of a parent state is established, Monte Carlo method is employed to make the sequence of internodes which is produced by child automaton to satisfy the statistical laws of parent state.

\section{INTELLIGENT GROWTH AUTOMATON}

The main data structure of IGA is bi-dimensional hash table (B-DHT) and bi-dimensional hash chain (B-DHC). The structure information of plant and physiology information of every organ are stored in B-DHT. IGA produces the plant and interacts with IPE by access and writing B-DHC.

\subsection{Definition of bi-dimensional hash table and bi- dimensional hash chain}

Definition 1(Phy-Hash Table): It is a hash table which stores the physiology information of metamer. At present, there are four parameters -$G C, N C, n$ and $F$. GC denotes the number of Growth Cycle from appearance to now. $N C$ denotes the number of Node Cycle from appearance to now. $n$ is the ordinal of child automaton in a cycle. The initial value of $G C, N C, n$ of 
active bud is 1 , while the initial value of dormant bud is the negative of the dormant period. The value of $F$ includes 0,1 and 2, that 0 represents the metamer is intercalary growth, while 1 represents the metamer is apical growth, and 2 represents the metamer and others in the branch is restrained.

Definition 2(Str-Hash Table): It is a hash table which stores the structure information of metamer. At present, there are three parameters --POS, ORI and Mesh. POS denotes the absolute position of the metamer and ORI denotes the absolute orientation in the plant. Mesh denotes the mesh graphics corresponding to an metamer, including color and size of the organs.

Definition 3(B-DHT): It is constituted of Phy-Hash Table and Str-Hash Table. If an internode has axillary buds, it is need to bracket the B-DHT of the axillary bud with '['and ']', and all B-DHTs of axillary buds are linked.

Definition 4(B-DHC): It is a chain which is constituted of all B-DHTs by link of a plant and mark $\Omega$. Two denotation ' $\{$ ' and ' $\}$ ' are added at the head and the end of $\Omega$ which represents the start and the end of the plant. Obviously, all of the branches of the plant are bracketed by '['and ']'.

Definition 5(Sequential B-DHC): It is a chain which is constituted of BDHTs without branch and mark $\delta$. For instance, the Sequential B-DHC of main stem is constituted of all B-DHTs which are inside ' $\{$ ' and ' $\}$ ' but outside '['and ']'.

\subsection{Modeling growth by intelligent growth automaton}

$\Omega$ is initialized according to axiom, and updated according to controlling information from 2DHA continuously, to generate a plant. Meanwhile, IGA gets the information of environment and functional organs, and modifies parameters of 2DHA to make plant growth accustomed to the environment.

$\delta$ represents a stem without branch. It have four possibilities: The first is that GC is less than 1, which means there is only one dormant bud; The second is that all GC equal 1, which means that all metamers appearance only this year; The third is all GC are greater 1, which means that all metamers appearance only before this year and restrained; The fourth is that the metamers appears this year and before.

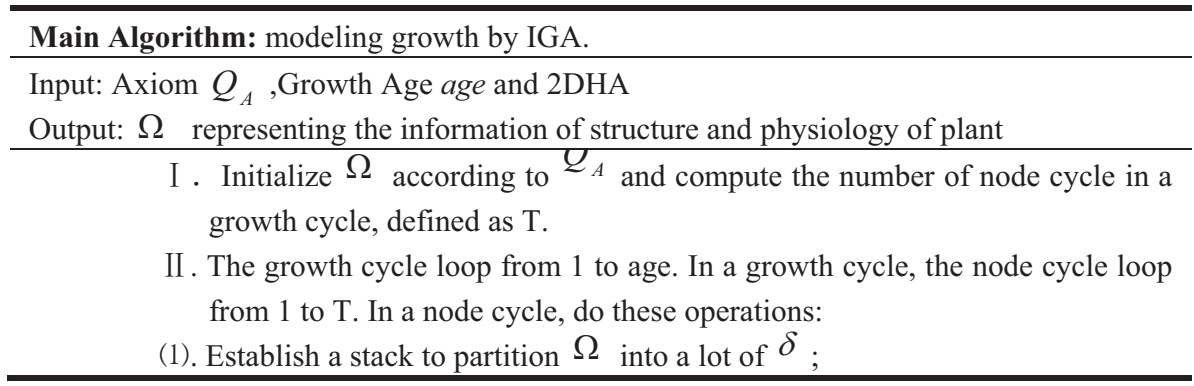


(2). Deal with every $\delta$;

(3). If the current $\Omega$ is processed successfully, plot $\Omega$. Compute the absolute position and orientation, size, height and other geometry information of every organ;

(4). Modify parameters of 2DHA according to IPE.

The above main algorithm depicts the process of growth modeling by IGA. The following child algorithm i, ii, iii are the detailed description of operation (1), (2), (4)in the main algorithm.

Child Algorithm i : the description of (1)--partition $\Omega$ into one by one $\delta$.

Input: $\Omega$

Output: $\delta$ (initial $\delta$ is null)

(1). Access every character from $\Omega$, mark ch from left to right;

(2). If $c h$ is B-DHT, or ' $\{$ ', '[', push $c h$ and go to 1); otherwise, pop to $y$;

(3). If $y$ is B-DHT, $y$ is added at the head of $\delta$; if $y$ is '[', an entire $\delta$ is acquired, go to main algorithm(2). When return from (2), $\delta$ initialize null and access the character after $c h$ from $\Omega$, go to (2); if $y$ is ' $\{, \Omega$ is processed successfully and go to main algorithm (3).

Child Algorithm ii : the description of (2)--deal with $\delta$.

Input: $\delta$

Output: modified B-DHT

(1). If the eldest metamer(the head) in $\delta$ is dormant, go to (2); if it appears this year, go to (3); if it appears before this year, go to (4);

(2). The $N C$ of this metamer plus 1 , if $G C$ is 0 , wakens it. Return;

(3). If the entire metamer sequence of this Growth Unit have already formed and this Growth Cycle is over, all GC of this Growth Unit plus 1, if Growth Cycle isn't over, the $N C$ of all metamers of this Growth Unit plus 1 . If the metamer sequence isn't completed, $n$ and $N C$ plus $1, F$ updated to 0 , select a new metamer according to the transfer matrix of the Child Automaton, add to $\Omega$, return;

(4). If the youngest metamer (the end) in $\delta$ appears this year, partition $\delta$ into $\delta_{1}$ (metamer sequence this year) and $\delta_{2}$ (metamer sequence before this year). Process $\delta_{1}$ with(3) and process $\delta_{2}$ with(5). When $\delta_{1}$ and $\delta_{2}$ are processed successfully, return. If the youngest metamer appears before this year, go to(5), after (5), return;

5. If the Growth Cycle is over, all GC of this Growth Unit plus 1, if the eldest metamer is restrained, return; if it isn't restrained, select a new metamer according to Parent Automaton and Child Automaton, add to $\Omega$, return. If Growth Cycle isn't over, return. 
Meanwhile, IGA modifies parameters of 2DHA by interacting with IPE, to make plant growth accustomed to environment dynamically. The function $f_{e}$ depicts IPE, which is defined as $f_{e}=F(\vec{e}, \vec{y})$, where $\vec{e}$ denotes all kinds of environment factor, such as illumination, water, accumulated temperature and mineral. $\vec{y}$ denotes all kinds of factor of organ function, such as age, height, branch, photosynthesis outcome and so on. The following child algorithm iii describes IPE impact on plant growth and development.

Child Algorithm iii: the description of (4)--modifies parameters of 2DHA.

Input: $f_{e}$

Output: 2DHA modified parameters

(1). If current $\Omega$ is processed successfully and Growth Cycle isn't over, modifies transfer matrix of Child Automaton according to function $f_{e} . F$ can be updated to 2 to make a metamer restrained, and the dormant period of bud can be modified;

(2). If Growth Cycle is over, modifies transfer matrix of Parent Automaton according to function $f_{e}$. And modifies $\pi_{c}, L_{c}$ and transfer matrix of Child Automaton of the next parent state.

\section{CONCLUSION AND DISCUSSION}

The growth and development of plant are controlled essentially by genes, and plant must grow in feasible environment. Plant absorbs mineral nutrition and energy from environment, and then grows and develops by material metabolism and energy metabolism. Therefore, genes and environment are the driving force of the growth and development of plant.

In the framework, 2DHA simulates the function of genetic information to control the branch pattern of plant and drives the growth and development of plant. IPE controls and coordinates the interaction between IAVOs which constitute the virtual plant, distribution and balance of carbohydrate inside plant, and the interaction between virtual plant and virtual environment, to drive the growth and development of plant, and display 3D graphics of plant. Therefore, the IGA employs genes and environment to control the growth and development of plant, and is capable of demonstrate the drive of genes and environment to the growth and development of plant vividly.

On the basis of IGA, a software tool which can plot the topology of plant has been developed to validate this model. The different growth in the normal and abnormal environment is simulated successfully through the software. Normal environment is a feasible environment for plant growth. While abnormal environment is harmful to plant living and growth, such as 

Engine

drought, low temperature, insect pest, and so on. In the experiment, abnormal environment is drought and low temperature. Fig. 3 shows the topology of four continuous plant growth phases in normal environment, while Fig. 4 shows the topology of plant growing in abnormal environment.

Experimental results show that the potential pattern of plant is controlled by genes, while the specific character of plant is affected by environment. In normal environment, the potential branch and organ will grow and develop, while abnormal environment make some buds dormant, restrained, dead or transform to other kind of tissue, Fig. 3 and Fig. 4 can illustrate this effect.

The genetic information can't be acquired directly by 2DHA. At present, complex statistical and clustering method are employed. At first, the plant is sampled and statistically computed, and then the genetic information is clustered and recognized. Therefore, new method must be employed, for example, the gene control network which is on the basis of plant physiology can be employed. In addition, it is worthy to do thorough research on the functional organ model and environment model, and the latest achievements in plant physiology should be employed to control the development of plant more vividly and effectively.

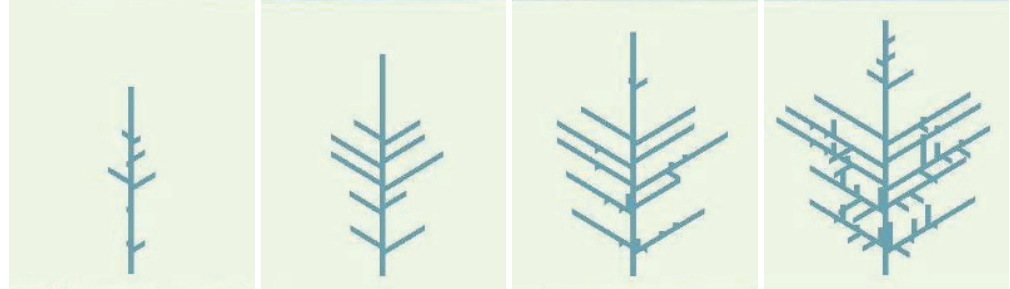

Fig.3 Normal growth

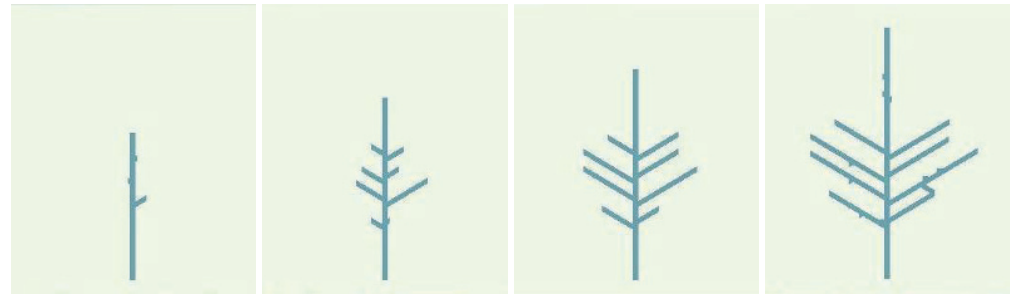

Fig.4 Abnormal growth

\section{ACKNOWLEDGEMENTS}

The authors are grateful to the National Natural Science Foundation of China (60773082) and the 863 program of China (2006AA10Z233). We are also grateful to Chongqing Academy of Agricultural Science of China who 
provided the plant physiological, morphological data for training and experimentations.

\section{REFERENCES}

F Blaise, J F Barczi, M Jaeger, P Dinouard, P De Reffye. Simulation of the growth of plants modeling of metamorphosis and spatial interactions in the architecture and development of plants. Cyberworlds, Tokyo: Springer-Verlag, 1998, 81-109

Hongchun Qu ,Qingsheng Zhu ,Qingqing Deng et al .Modelling and Consructing of Intelligent Physiological Engine Merging Artificial Life for Virtual Plants. Journal of Computational and Theoretical Nanoscience, 2007 (4) pp.1405-1411

Kyle W. Tomlinson et at. A functional-structural model for growth of clonal bunchgrasses. ecological modelling, 2007 (202) 243-264.

Li Hesheng. Modern Plant Physiology. Higher Education Press, Beijing, 2002

P Prusinkiewice, A Lindenmayer. The algorithmic beauty of plants. New York: SpringerVerlag, 1990

S Dekmo, L Hodges, B. C Naylor onstruction of fractal objects with iterated fuction systems. Computer Graphics, 1985, 19(3): 271-278

W Reeves. Approximate and probabilistic algorithms for shading and rendering structured Particle Systems. Computer graphics, 1985, 19(3): 313-322

X G Viennot, G Eyrolles, N Janey, D Arques. Combinatorial analysis of ramified patterns and computer imagery of trees. Computer graphics, 1989, 23(3): 31-39

Yang Shijie. Plant Biology. Science Press, Beijing, 2000.

Zhao Xing, Ph de Reffye, Xiong Fan-Lun et al . Dual-scale automaton model for virtual plant development. Chinese Journal of Computers , 2001 ,24 (6) :608 - 617 (in Chinese) 